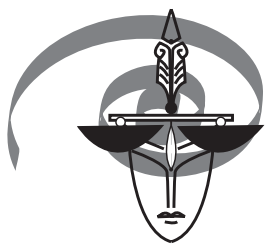

\title{
EUROPEAN
}

Volume $8 \cdot 2014 \cdot$ Number 2 (28)

DOI: 10.2478/ep-2014-0008

Donald J. Krapohl" American Polygraph Association

\section{Letter to the Editor: Calculating Polygraph Decision Accuracy}

A recent article in European Polygraph caught my attention for the manner in which polygraph decision accuracy was calculated (Patton, 2013). In brief, from a sample of 151 cases drawn over five years, the author reported he had 143 true positives, 6 inconclusives, and two false positives. Accuracy was reported in two ways, once with inconclusives counted as errors, and with inconclusives removed. The author reported $95 \%$ accurate decisions for the former, and $98 \%$ for the latter.

In the mainstream literature, polygraph decision accuracy is reported with and without inconclusives, as Patton (2013) had done. One difference, though, is how overall accuracy is calculated. The conventional approach is to average the decision accuracy between deceptive and truthful cases. The formula is below:

$\left(\frac{\text { True Positives }}{\text { (True Positives }}+\frac{\text { True Negatives }}{\text { (True Negatives }+ \text { False Positives) })}\right)$

"The author is a member of the American Polygraph Association, and the Editor of its publications. Comments welcome at APAkrapohl@gmail.com. 
In plain language, this formula calculates the accuracy for truthful and deceptive cases separately, then takes the average of those two accuracies. For example, if a polygraph technique were $80 \%$ accurate with truthful cases, and $92 \%$ accurate with deceptive cases, setting aside inconclusives, the overall accuracy of that technique would be $86 \%$, that being the average of $80 \%$ and $92 \%$.

Returning to the Patton (2013) data, and excluding inconclusives the technique caught all of the deceptive examinees in the sample, but the two false positives show that it detected none of the truthful cases. Averaging the $100 \%$ accuracy with the deceptive cases with the $0 \%$ accuracy with the, albeit, limited number of truthful cases produces an accuracy of $50 \%$. The difference between the 98\% accuracy reported in Patton (2013) and the 50\% estimate for the standard method is substantial, and worthy of comment.

By way of illustration, suppose that a researcher in the field collected a sample of 100 cases. All of them had been called DI and there had been a posttest confession to confirm it. The sample had no confirmed truthful cases. Would it be correct to conclude the polygraph technique was 100\% accurate? The short answer is no, because the actual accuracy of the polygraph could not be calculated with only these data. Without determining the accuracy of the technique in detecting both deception and truthfulness, the findings would be meaningless. It might be that the technique can detect $100 \%$ or $50 \%$ or none of the truthful cases. and the final accuracy estimate will depend on which figure it is. Without knowing the number would preclude a calculation for accuracy. This is one reason the standard formula became the standard.

Another challenge to the paper can come from the exclusive use of the confession criterion for comparing against polygraph decisions. The problem is that it can produce a non-representative sample that works in favor of high accuracy. Consider this: if the polygraph results are DI, there will be an interrogation, and deceptive examinees are more likely to confess if interrogated. Conversely, when the results are NDI (right or wrong) there is no interrogation and consequently no confession. If only confession cases are selected, they will be only those where there was an interrogation. Non-confessing true positives are indistinguishable from non-confessing false negatives, both of which come with DI results and no confession. False negative and false positive errors are not easily detected using only confessions as the basis for inclusion in a sample. From the Patton sample, 
one could argue that calling every case DI with whatever technology would lead to the exact same accuracy as did the polygraph: all deceptive cases were correctly identified, and none of the truthful cases.

In fairness I would like to make clear that I am not contending the technique used by Patton is $50 \%$ accurate, nor that the Patton sample was intentionally biased. Rather, the purpose is to point out two oft-encountered pitfalls in polygraph efficacy research, sampling and statistical methodology, and to counsel readers and writers to be mindful of their impact on research results. While Patton's paper is clearly instructive in what it says about the utility of his polygraph technique as regards securing confessions, the statistical information concerning decision accuracy oversteps what the methodology and data can support.

\section{References}

Patton, C.A. (2013). The Integrated Zone Comparison Technique: A field utility study in a deceptive population. European Polygraph, 7(3), 113-120. 